\title{
Vitamin D, Zinc and Iron in Adult Patients with Covid-19 and Their Action in the Immune Response as Biomarkers
}

Eliza Miranda Ramos ${ }^{1,2^{*}}$, Emerson Luiz Lima Araújo ${ }^{3}$, Francisco José Mendes dos Reis ${ }^{1,2}$, Igor Domingos de Souza $^{1,2,5}$, Gilberto Gonçalves Facco ${ }^{4}$, Iara Barbosa Ramos ${ }^{2}$, Pamella Aline Miranda Teodoro ${ }^{2}$, Antônio Carlos de $\mathrm{Abreu}^{2}$, Alessandro Carvalho da Fonseca ${ }^{2}$, Ernani Mendes da Fonseca Junior ${ }^{5} \&$ Valter Aragão do Nascimento ${ }^{1,2^{*}}$

1 Group of Spectroscopy and Bioinformatics Applied to Biodiversity and Health, School of Medicine, Postgraduation Program in Health and Development in the Midwest Region, Faculty of Medicine, Federal University of Mato Grosso do Sul, Campo Grande, Mato Grosso do Sul, Brazil

${ }^{2}$ Postgraduation Program in Health and Development in the Midwest Region, Faculty of Medicine, Federal University of Mato Grosso do Sul, Campo Grande, Mato Grosso do Sul, Brazil

3 Ministry of Health, Secretary of Health Surveillance, Department of Strategic Coordination of Health Surveillance, General Coordination of Public Health Laboratories, Brasília-DF, Brazil

${ }^{4}$ Graduate Program in Environments and Regional Development, Anhanguera University, UNIDERP, Mato Grosso do Sul, Brazil

${ }^{5}$ Estácio de Sa University, Campo Grande, Mato Grosso do Sul, Brazil

Correspondence: Eliza Miranda Ramos \& Valter Aragão do Nascimento, Group of Spectroscopy and Bioinformatics Applied to Biodiversity and Health, School of Medicine, Postgraduation Program in Health and Development in the Midwest Region, Faculty of Medicine, Federal University of Mato Grosso do Sul, Campo Grande, Mato Grosso do Sul, Brazil.

* Contributed equally.

Received: May 31, 2021 Accepted: September 18, 2021 Online Published: November 15, 2021

doi:10.5539/gjhs.v14n1p1 URL: https://doi.org/10.5539/gjhs.v14n1p1

\begin{abstract}
COVID-19 in 2020 brought challenges to the Brazilian public health system with an emerging virus with respiratory contagion called SARS-CoV-2. There are few studies in Brazil and in some countries, on the increased incidence of certain viral respiratory infections, including H1N1 and coronavirus and their association with low levels of vitamin D, zinc and iron. The aim of this study was to demonstrate that the deficit of vitamin D, zinc and iron has an impact on the infectious process of patients with COVID-19 and to establish new forms of prevention for the worsening of COVID-19 in the human body. Data were collected from medical records and test results from patients being followed up during the treatment period for COVID-19. Patients with low blood levels of vitamin D, zinc and iron during the treatment period of COVID-19 had a higher percentage of worsening and complications requiring hospitalization in intensive care beds. The ingestion of vitamin $\mathrm{D}$, zinc and iron in the treatment period of patients with COVID-19 in addition to being an immunological protector against SARS-CoV-2 and alleviating the process of worsening the disease can also act as a biomarker in cases of this disease.
\end{abstract}

Keywords: Zinc, vitamin D, iron, gripes, COVID-19, anemia

\section{Introduction}

In April 2020, Brazil began to mobilize to combat COVID-19 in order to establish risk factors and prognostic markers, such as the deficit of Vitamin D, zinc and iron (OPAS, OMS. 2020; Ramos, Mendes dos Reis, de Souza, de Freitas, de Lima, \& de Abreu, 2020). COVID-19 reached Brazilian states such as São Paulo, Rio de Janeiro, Fortaleza and Amazonas, and in adult infected patients the result was the development of severe pneumonia through Beta Coronavirus (SARS-COV-2) (OPAS, OMS, 2020; Ramos, E. M., Mendes dos Reis, Ramos, M. V., de Souza, \& Bochenek, 2020). However, cases of death in Brazil have characteristics related to viral pathogenicity without worsening the patient according to a deficit of macro and micro elements (Ramos, Mendes dos Reis, de Souza et al., 2020; Ramos, E. M., Mendes dos Reis, Ramos M. V. et al., 2020; Razzaque, 2020).

COVID-19 patients in Brazil are adults with one or more comorbidities (Xu et al., 2020; Zhou et al., 2020). Most 
patients are over 50 years old and have pre-existing diseases mainly in the heart and respiratory system; some patients are smokers, which cause complications (Ramos, de Abreu, et al., 2020; Ramos, Mendes dos Reis et al., 2020). On the other hand, there are patients who have severe kidney diseases that hinder clinical improvement during the treatment period (OPAS, OMS, 2020; Ramos, Mendes dos Reis, de Souza, et al., 2020). In addition, hypertension or chronic and neurodegenerative diseases brings aggravations such as death during the treatment period (Zhou et al., 2020).

In Italy, COVID-19 manifests itself in patients through high fever $\left(\geq 38.0^{\circ} \mathrm{C}\right)$, fatigue, non-productive cough, dyspnea and diarrhea. In this case, laboratory tests of patients with confirmed diagnosis show normal or reduced white blood cell count, as well as reduced lymphocyte count, especially in severe cases, thrombocytopenia, high transaminase, high lactate dehydrogenase (LDH), high creatine kinase (CK) and myoglobin elevated (Ramos, de Abreu, de Freitas et al., 2020; Prasad, Bao, Beck, \& Sarkar, 2011; Ramos, Mendes dos Reis, de Souza et al., 2020). The death of patients occurs in severe cases, because the disease progresses quickly to septic shock with great difficulty in treatment due to the presence of metabolic acidosis and coagulation dysfunction (Razzaque, 2020; Prasad et al., 2011).

Studies in Brazil, China, Italy and the United Kingdom involving Covid-19 have brought direct primary outcomes of this disease with comorbidities, mainly responses to direct primary immune deficiency such as the body's immune deficiency, coagulation, coagulative activation, myocardial injury, liver damage, kidney damage and secondary bacterial infectious correlations (Malek Mahdavi, 2020; Mohammad, Mishra, \& Ashraf, 2019; Xu et al., 2020). The virus reaches the innate immune system through non-specific responses whereas in the adaptive system the responses are specific to the antigen by the innate immune response (Huang et al., 2020). However, in Brazilian patients it is possible to verify that severe cases with evolution to death tend to occur in patients who have developed lymphopenia and secondary inflammation (Cen et al. 2020; Giacomelli et al., 2020).

In addition to the text quoted above, there are cases of patients who are able to recover from Covid-19 with little or no medical and hospital intervention, in this case, patients who do not have deficits in elements such as zinc, iron and Vitamin D (Chan et al., 2020; Grant et al., 2020; Read et al., 2020). Thus, this discrepancy as an immunological outcome of patients with COVID-19 has challenged the Brazilian public health system to find an adequate response (D'Avolio et al., 2020; Huang et al., 2020). Some Brazilian regions have limited data, which makes it difficult to make statements about populations that may be susceptible to COVID-19 (Ampawong et al., 2015; Gruber-Bzura, 2018). However, it is possible to state that the severity of the disease is associated with some underlying conditions of the host, such as age, biological sex, immunological health, deficits of macro- and microelements, vitamin D, zinc and iron and the existence of diseases pre-existing such as diabetes (Cen et al., 2020).

Given the above, the objective of this study was to describe through a case report that the deficit of vitamin D, zinc and iron has an impact on the infectious process of patients with COVID-19 and on the worsening process, as well as on the development of complications in COVID-19 during the treatment period.

\section{Materials and Methods}

\subsection{Study Population}

In this case report, patients aged $\geq 40$ years and with laboratory diagnosis of COVID-19 through real-time reverse transcriptase "polymerase chain reaction assay (RT-qPCR)" for specific severe acute respiratory tracts were included in the positive control groups (Treatment).

In addition, patients with blood tests with dosages of elements such as zinc, iron and levels of vitamin D (25-hydroxyvitamin D $(25(\mathrm{OH}) \mathrm{D})$ who presented deficits in the results were included, and those patients who presented consistent symptoms for COVID-19 such as cough, dyspnoea, fever, fatigue and loss of smell and taste in the period from June to October 2020.

All patients were observed by public health professionals and private hospitals during the period of preventive treatment to control worsening by chest X-ray or chest and D-dimer tomography. Patients who did not show any deficits in the results were included in the negative control group for COVID-19.

The positive control group for COVID-19 was divided into patients with vitamin D, zinc and iron deficiency. In the positive control group (Treatment) with Vitamin D deficit, patients were included according to national and international guidelines with local laboratory standards (Vitamin $\mathrm{D} \leq 20 \mathrm{ng} / \mathrm{dL}$ ), zinc ( $\mathrm{Zn} \leq 70 \mathrm{ug} / \mathrm{dL}$ ).

The positive control group or treatment group for COVID-19 was divided into patients with vitamin $\mathrm{D}$, zinc and iron deficiency. In the positive control group (Treatment) with Vitamin D deficit, patients were included according 
to national and international guidelines with local laboratory standards (Vitamin $\mathrm{D} \leq 20 \mathrm{ng} / \mathrm{dL}$ ), zinc ( $\mathrm{Zn} \leq 70$ $\mathrm{ug} / \mathrm{dL})$.

In addition, an evaluation of the blood iron level was performed in the laboratories of the Brazilian public health system or private health system with the purpose of verifying the presence of anemine, verifying the deficit of serum ferritinin and serum iron to be used as a determinant of health or worsening of the patient's health with COVID-19. The treatment group included adult participants who had $\mathrm{Hb}$ levels $\leq 11 \mathrm{ng} / \mathrm{dL}$ and serum iron levels $<50 \mathrm{ug} / \mathrm{dL}$ according to the standard used by the Ministry of Health in Brazil. The treatment of patients with CO-VID-19 in public or private hospitals was carried out in accordance with the national guidelines and care instituted by the Ministry of Health of Brazil. Some patients were treated with subcutaneous low molecular weight heparin as a prophylaxis of venous thromboembolism according to guidelines from the Ministry of Health of Brazil.

\subsection{Data Collection Methods}

In this experimental and observational study, data were collected through the extraction of medical records and results of follow-up exams in hospitals and laboratories with a database of patients included in the study. Also, age, weight, BMI, smoking and hyperglycemia were included. COVID-19 related hospital mortality was considered as the primary outcome.

Results that included patients with alterations in radiological images and with the presence of important cellular laboratory alterations were considered as results. In order to maintain the quality of clinical care, Vitamin D, zinc and iron were quantified as requested during the treatment period at the care institution. The data were stored in an Excel spreadsheet which contains biochemical and hematological information. The entire procedure was carried out according to the Brazilian guidelines for the diagnosis and prognosis of COVID-19 through the measurement of C-reactive protein (CRP), D-dimer, ferritinin, hemoglobin, high sensitivity of troponin T, lactate dehydrogenase (LDH) and count of lymphocytes and other cells of the defense system in the human body. The data related to the cause of death was obtained by verifying the death certificates issued by hospital institutions held by family members.

\subsection{Ethical Principles}

This research was authorized by the Department of Continuing Education of the city of Campo Grande/MS and the Federal University of Mato Grosso do Sul for data collection. Clinical data were collected and were not identifiable.

All data were collected in situ and treated according to the regulations of the Ethics Committee of the Federal University of Mato Grosso do Sul considering local standards on data protection (CAAE: 42969320.0.0000.0021). All practices carried out in the study were carried out in accordance with regulations and clinical practice protocols. Serum levels of Vitamin D, zinc and iron were performed at the health institution to which the patient attended for the treatment of COVID-19 and did not require researchers to perform phlebotomy and supplementation of zinc, iron and vitamin was not performed. D. All patients were monitored and received care according to standard practice for the treatment of COVID-19 over the period of the study offered by the Ministry of Health in Brazil.

\section{Results}

A total of 20 patients aged 40 to 80 years were selected in this study. Only 8 men and 5 women totaling 13 patients $(65 \%)$ with a positive result for COVID-19 were allocated to the "positive control or treatment" group, on the other hand, 7 patients (35\%) without a diagnosis of COVID-19 were allocated to the "negative control" (Table 1).

In the positive control group, 9 patients $(45 \%)$ had a level of $25(\mathrm{OH}) \mathrm{D} \leq 20 \mathrm{ng} / \mathrm{dL}$ and 02 patients $(10 \%)$ had a level greater than $20 \mathrm{ng} / \mathrm{dL}$ (Table 3). In addition, 8 patients (61.5\%) had a level of Zinc (Zn) $\leq 70 \mathrm{ug} / \mathrm{dL}$ and 1 patient (7.6\%) had a level greater than $120 \mathrm{ug} / \mathrm{dL}$ (Table 3).

When assessing the blood level of iron concentration in the positive control group that underwent treatment, it was found that 9 patients $(69.2 \%)$ had a serum iron level equivalent to $<50 \mathrm{ug} / \mathrm{dL}$. On the other hand, 5 female patients $(38.4 \%)$ also had a ferritinin blood level $<23.6 \mathrm{ng} / \mathrm{mL}$. However, 4 patients $(30.7 \%)$ were male and had ferritinin $<$ $11.0 \mathrm{ng} / \mathrm{ml}$, of all patients included in the treatment group, a total of 8 patients $(61.5 \%)$ had hemoglobin $<11(\mathrm{~g} / \mathrm{L})$ (Table 3$)$. In addition, 8 patients $(61.5 \%)$ were included in the treatment group are diabetics with glucose $>99$ $\mathrm{mg} / \mathrm{dL}$ (Table 3) in blood level.

Based on the results of the 13 patients belonging to the treatment group (Positive control group) with COVID-19, it is possible to verify that about 9 patients $(69.2 \%)$ had a hemoglobin level lower than that standardized in Brazil (Reference value of $11-15 \mathrm{ng} / \mathrm{dL}$ ). It was found that these lower hemoglobin levels occurred in patients over the 
median age (60 years) and with a greater proportion in female $(\mathrm{n}=8)$ and diabetic $(61.5 \%)$ patients. In addition, these diabetic patients with low hemoglobin levels worsened during the COVID-19 treatment period and needed to be admitted to intensive care unit beds (Table 3 and Table 2).

A total of 9 patients in the treatment control group (69.2\%) had D-dimer with elevation levels above $1 \mathrm{mcg} / \mathrm{ml}$. On the other hand, 5 patients (38.4\%) progressed to mortality during the hospitalization period with a hospital stay of more than 20 days, which mainly evolved to radiological worsening (Table 2 and Graph 2).

The clinical data of positive control patients with COVID-19 and with vitamin D, zinc and iron deficiency (Temperature, $\mathrm{SaO} 2$, hospitalization, Use of intensive therapy with use of mechanical ventilation and death) were compared with the negative control groups (Table 2). From the comparisons between groups, it was possible to verify that 2 patients in the positive control group (15.3\%) used intensive care and had the need to use mechanical ventilation, however, in the negative control group 6 patients $(46,1 \%)$ experienced the need to use intensive therapy with the use of mechanical ventilation (Table 2 and Graph 1). Thus, it is possible to verify that there was a difference in the mean length of stay of patients in the positive control group (treatment) with COVID-19 during the hospitalization period compared to the negative control without COVID-19 (Table 2).

Blood levels of vitamin $\mathrm{D}$ in the positive control group (treatment) with COVID-19 were significantly lower than the reference values used in Brazil, which totaled 9 patients (69.2\%) (Table 3). The zinc concentration was lower in the positive control group (treatment) with COVID-19 compared to the standardized reference value in Brazil, and totaled 8 patients $(61.5 \%)$. Serum iron, ferritinin and hemoglobin were lower than the values standardized by Brazil, totaling 9 patients in the positive control group with COVID-19 (69.2\%) (Table 3).

According to the results, 7 patients had a vitamin D deficit (53.8\%), as well as 7 patients had a zinc deficit (53.8\%). On the other hand, 9 patients had an iron deficit (45\%). An interesting fact is that such patients with vitamin D, zinc and serum iron deficits evolved with worsening in COVID-19 and had a high average in the level of D-dimer. Such patients required hospitalization with the use of the bed in intensive care and mechanical ventilation during the treatment period. In addition, 5 patients progressed to death, mainly those with pulmonary worsening (Table 3 , Graph 1 and Graph 2).

In the positive control group with COVID-19, patients with Vitamin D, zinc and iron deficiency had higher peak levels of lymphocytes, leukocytes, Natural Killers cells, IL-6, IL-4, IL-10, IL-2 and IL-8 due to the increase in the hospitalization period and the need to use intensive care (Table 2, Table 3 and Graph 1). In this case, it was observed that these patients showed an increase in the percentage of peak levels in CD3 (7,35\%). It is possible to verify a significant difference in mortality in the positive control group with COVID-19 when compared with the negative control group COVID-19 (Table 2).

Table 1. Demographic characterization of patients with COVID-19 according to the case report

\begin{tabular}{|c|c|c|c|c|c|c|}
\hline \multicolumn{7}{|c|}{ DEMOGRAPHIC DATA } \\
\hline Age & Male & Female & Tobacco Use & $\begin{array}{l}\text { IMC } \geq 30.0 \\
\text { a } 39.9\end{array}$ & $\begin{array}{l}\text { Patients with } \\
\text { Covid-19 Positive } \\
\text { Control Group or } \\
\text { treatment }\end{array}$ & $\begin{array}{l}\text { Patients without } \\
\text { COVID-19 Negative } \\
\text { Control Group }\end{array}$ \\
\hline$(40-50)$ & 1 & 1 & 1 & 2 & 2 & 2 \\
\hline$(50-60)$ & 2 & 1 & 1 & 2 & 3 & 2 \\
\hline$(60-70)$ & 3 & 1 & 3 & 4 & 4 & 1 \\
\hline$(70-80)$ & 2 & 2 & 1 & 1 & 4 & 2 \\
\hline TOTAL & & & & & 13 & 07 \\
\hline
\end{tabular}


Table 2. Clinical characterization of patients with COVID-19 according to a case report

\section{CLINICAL DATA}

\begin{tabular}{lllll}
\hline Clinical Data & Male & Female & $\begin{array}{l}\text { Patients } \\
\text { Positive - Control Group(n= } \\
\text { 13) }\end{array}$ & $\begin{array}{l}\text { Patients without COVID-19 } \\
- \text { Negative Control Group(n } \\
=7)\end{array}$ \\
\hline Temperature $\left({ }^{\circ} \mathrm{C}\right)$ & 2 & 1 & 2 & 2 \\
\hline$(36.0-37.9)$ & 2 & 2 & 8 & 2 \\
$(38.0-38.5)$ & 4 & 1 & 3 & 3 \\
$(38.5-39.5)$ & 1 & & & 2 \\
\hline $\mathrm{SpO} 2$ & 4 & 2 & 6 & 2 \\
\hline$(91 \%-100 \%)$ & 1 & 0 & 1 & 1 \\
$(81 \%-90 \%)$ & 0 & 1 & 1 & 0 \\
$(71 \%-80 \%)$ & 2 & 1 & 3 & 2 \\
$(61 \%-70 \%)$ & & & & \\
$(50 \%-60 \%)$ & & & & \\
\hline
\end{tabular}

Hospitalization (Days hospitalized)

$\begin{array}{lllll}(01-10) & 1 & 1 & 2 & 7 \\ (11-20) & 1 & 1 & 2 & 0 \\ (21-30) & 3 & 2 & 5 & 0\end{array}$

Use of intensive therapy using mechanical ventilation (Days)

$\begin{array}{lllll}(01-10) & 1 & 1 & 2 & 1 \\ (11-20) & 1 & 1 & 2 & 0 \\ (21-30) & 1 & 1 & 2 & 0\end{array}$

Death during the patient's follow-up period in the case report

Death during the patient's

follow-up period in the case 3

report 
Table 3. Characterization of the laboratory results of patients with COVID-19 according to the case report

\begin{tabular}{|c|c|c|c|c|}
\hline \multicolumn{5}{|c|}{ RESULTS OF LABORATORY EXAMINATIONS } \\
\hline Clinical Data & $\begin{array}{l}\text { Male } \\
\text { N (\%) }\end{array}$ & $\begin{array}{l}\text { Female } \\
\text { N (\%) }\end{array}$ & $\begin{array}{l}\text { Patients with } \\
\text { Covid-19 - Positive } \\
\text { Control Group - N } \\
(\%)(n=13)\end{array}$ & $\begin{array}{l}\text { Patients } r \text { without } \\
\text { COVID-19 - Negative } \\
\text { Control Group }- \text { N ( \%) (n } \\
=7)\end{array}$ \\
\hline \multicolumn{5}{|l|}{ Serum Vitamin D (ng/dL) } \\
\hline$<\mathbf{2 0}$ & 5 & 4 & 9 & 2 \\
\hline$(20-60)$ & 1 & 1 & 2 & 2 \\
\hline$\geq 100$ & 1 & 1 & 2 & 3 \\
\hline \multicolumn{5}{|l|}{ Serum Zinc (ug/dL) } \\
\hline$<70$ & 5 & 3 & 8 & 3 \\
\hline$(70.0-120)$ & 3 & 1 & 4 & 2 \\
\hline$>120$ & 0 & 1 & 1 & 2 \\
\hline \multicolumn{5}{|l|}{ Serum Iron (ug/dL) } \\
\hline$<\mathbf{5 0}$ & 4 & 5 & 9 & 1 \\
\hline $50-175$ & 0 & 1 & 1 & 3 \\
\hline$>175$ & 2 & 1 & 3 & 3 \\
\hline \multicolumn{5}{|l|}{ Ferritin (ng/mL) } \\
\hline$>23.9$ (Male reference value) & 4 & 0 & 4 & 5 \\
\hline$<23.9$ (Female reference Value) & 0 & 5 & 5 & 2 \\
\hline \multicolumn{5}{|l|}{ Hemoglobin $(g / L)$} \\
\hline$<11$ & 1 & 8 & 9 & 2 \\
\hline$(11-15)$ & 2 & 1 & 3 & 2 \\
\hline$>15$ & 0 & 1 & 1 & 3 \\
\hline \multicolumn{5}{|l|}{ Protein C (\%) } \\
\hline 1.0 - $3.0 \mathrm{mg} / \mathrm{L}$ & 3 & 6 & 9 & 7 \\
\hline \multicolumn{5}{|l|}{ Proteín S (\%) } \\
\hline $67.5-139.0 \%$ (Male reference value) & 5 & 0 & 5 & 7 \\
\hline $60.1-113.6 \%$ (Female reference Value) & 0 & 4 & 4 & 7 \\
\hline \multicolumn{5}{|l|}{ Glycated Hemoglobin (Hb A1c) } \\
\hline $5.7 \%-6.4 \%$ & 2 & 03 & 5 & 5 \\
\hline$\geq 6.4 \%$ & 3 & 05 & 8 & 2 \\
\hline \multicolumn{5}{|l|}{ D-Dimer ( mcg/mL) } \\
\hline$<0.5$ & 2 & 2 & 4 & 5 \\
\hline$>1$ & 5 & 4 & 9 & 2 \\
\hline \multicolumn{5}{|l|}{ Glucose (mg/dL) } \\
\hline $70-99$ & 2 & 3 & 5 & 4 \\
\hline$>99$ & 3 & 5 & 8 & 3 \\
\hline \multicolumn{5}{|l|}{ Auxiliary T lymphocytes CD4+ (\%) } \\
\hline$<30.7$ & 1 & 1 & 2 & 4 \\
\hline $30.7-49.0$ & 8 & 1 & 9 & 2 \\
\hline$>49.0$ & 1 & 1 & 2 & 1 \\
\hline
\end{tabular}




\section{Total Leukocytes (cells / $\mu \mathrm{L}$ )}

\begin{tabular}{|c|c|c|c|c|}
\hline$<3.8000$ & 3 & 1 & 4 & 4 \\
\hline $3.800-11.000$ & 1 & 1 & 2 & 1 \\
\hline$>11.000$ & 3 & 4 & 7 & 2 \\
\hline \multicolumn{5}{|c|}{ CD4 T lymphocytes $($ CD3 + CD4 +) $($ cells $/ \mu \mathrm{l})$} \\
\hline$<477$ & 4 & 5 & 9 & 4 \\
\hline $477-1140$ & 1 & 1 & 2 & 1 \\
\hline$>1140$ & 1 & 1 & 2 & 2 \\
\hline \multicolumn{5}{|c|}{ Total Lymphocytes (cells / $\mu \mathrm{l}$ ) } \\
\hline$<1000$ & 1 & 1 & 2 & 4 \\
\hline $1000-4000$ & 1 & 8 & 9 & 1 \\
\hline$>4000$ & 1 & 1 & 2 & 2 \\
\hline \multicolumn{5}{|c|}{ Cytotoxic T lymphocyte CD8 + (CD8 (CD3 + CD8 + / CD3 +)\% } \\
\hline$<13.8$ & 0 & 1 & 1 & 4 \\
\hline $13.8-27.4$ & 5 & 5 & 10 & 1 \\
\hline$>27.4$ & 1 & 1 & 2 & 2 \\
\hline
\end{tabular}

CD8 T lymphocytes (CD3 + CD8 +) (cells / $\mu \mathrm{L})$

\begin{tabular}{|c|c|c|c|c|}
\hline$<211$ & 1 & 1 & 2 & 4 \\
\hline $211-724$ & 3 & 5 & 8 & 1 \\
\hline$>724$ & 2 & 1 & 3 & 2 \\
\hline \multicolumn{5}{|l|}{ CD3 \% } \\
\hline$<55.5$ & 2 & 5 & 7 & 4 \\
\hline $55.5-75.2$ & 1 & 2 & 3 & 1 \\
\hline$>75.2$ & 1 & 2 & 3 & 2 \\
\hline \multicolumn{5}{|c|}{ Natural Killer cells $($ CD56 + CD16) $($ cells $/ \mu)$} \\
\hline$<137$ & 1 & 2 & 3 & 4 \\
\hline $137-567$ & 2 & 5 & 7 & 1 \\
\hline$>567$ & 1 & 2 & 3 & 2 \\
\hline \multicolumn{5}{|c|}{ Interleukin 6 (IL-6) (pg/mL) } \\
\hline$>5.9$ & 5 & 5 & 10 & 3 \\
\hline \multicolumn{5}{|c|}{ Interleukin 10 (IL-10) (pg/mL) } \\
\hline$<9.1$ & 7 & 4 & 11 & 3 \\
\hline \multicolumn{5}{|c|}{ Interleukin 2 (IL-2) $\quad(\mathrm{UI} / \mathrm{mL})$} \\
\hline$<0.10$ & 6 & 4 & 10 & 3 \\
\hline \multicolumn{5}{|c|}{ Interleukin 4 (IL-4) (pg/mL) } \\
\hline$<38.7$ & 6 & 5 & 11 & 3 \\
\hline \multicolumn{5}{|c|}{ Interleukin 8 (IL-8) (pg/mL) } \\
\hline$<\mathbf{5 0 . 0}$ & 7 & 2 & 9 & 3 \\
\hline \multicolumn{5}{|c|}{ Neutrophils $(\mu \mathrm{L})$} \\
\hline$<1600$ & 1 & 1 & 2 & 4 \\
\hline $1600-8000$ & 1 & & 2 & 1 \\
\hline$>8000$ & 4 & 5 & 9 & 2 \\
\hline
\end{tabular}




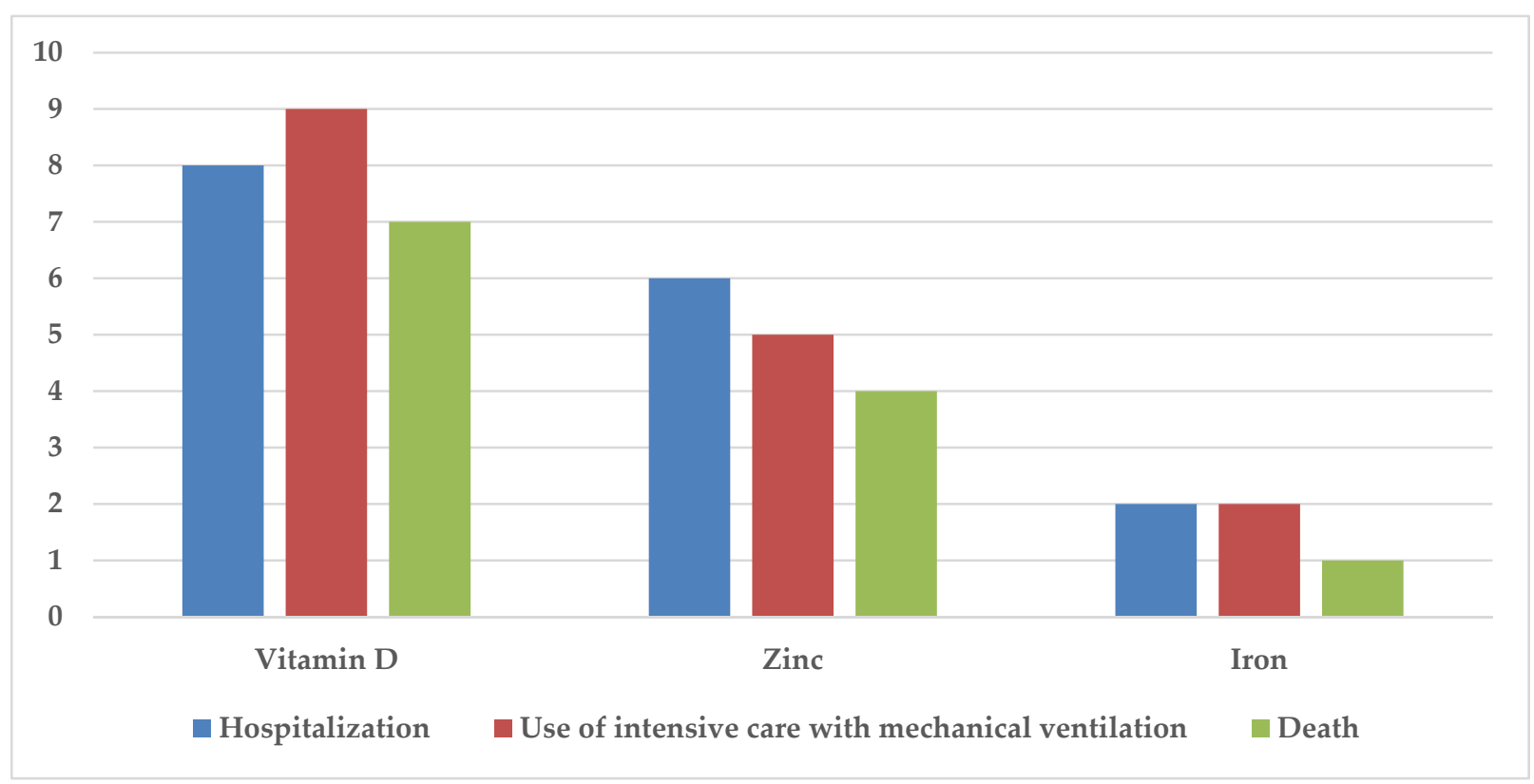

Graph 1. Deficit of Vitamin D, Zinc and Iron as biomarkers in the clinical worsening in patients with COVID-19

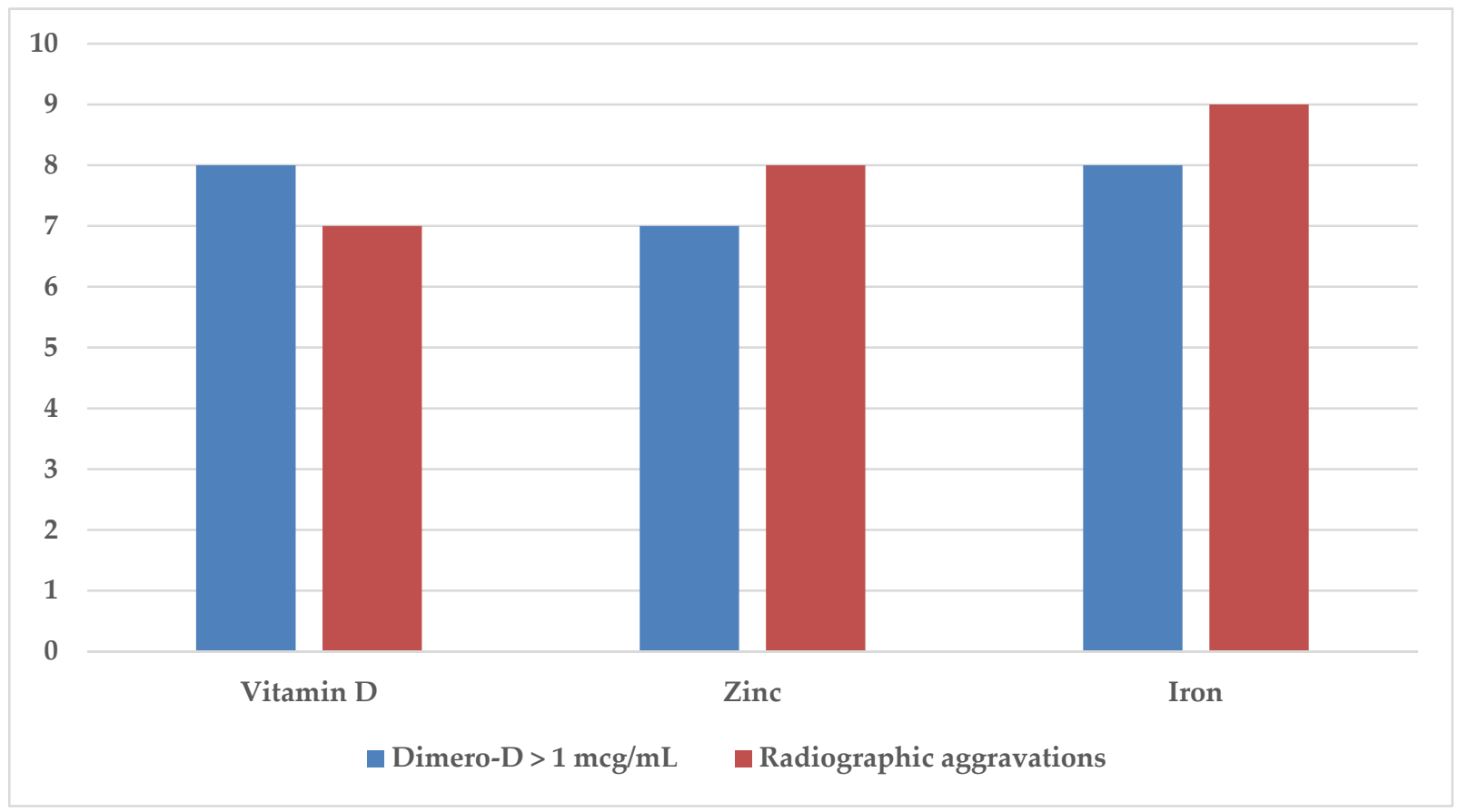

Graph 2. Characterization of Vitamin D, Zinc and Iron deficiency as biomarkers in radiographic deterioration and D-dimer $>1 \mathrm{mcg} / \mathrm{mL}$ in patients with COVID-19

\section{Discussion}

In this case report study, it is possible to verify that when adjusting the data in a linear regressive manner, a significant trend is observed for mean hemoglobin and serum iron at levels that were below the standard and that are related to the average age of 60 years of the participants in the study (Table 1 and Table 2) (International Artemisinin Study Group, 2004; Anstey et al., 2002; Ramos, de Abreu et al., 2020). Compared to other studies with patients who presented moderate symptoms in COVID-19, it is possible to observe that there is a similarity 
between both, because the patients with severe symptoms in this study also had the lowest hemoglobin levels when used as a standard (Alves et al., 2007; Ampawong et al., 2015; Ramos et al., 2020). In addition, hemoglobin and iron levels decreased with a higher percentage in diabetics, which was similar to other studies already developed (Brenner, Holleczek, \& Schöttker, 2020; Bishop et al., 2021; Ramos, de Abreu et al., 2020; Ramos, E. M., Mendes dos Reis, F. J., Ramos, M. V., et al., 2020).

Huang et al. (2020) in their descriptive study obtained similar data in which hemoglobin levels in $45 \%$ of patients with COVID-19 were hospitalized and required an intensive care bed (Anstey et al., 2002; Ramos, E. M., Mendes dos Reis, Ramos, M. V., 2020). The results presented in our study corroborate the study carried out by Wang et al (2020), which demonstrated a worsening in the symptoms of patients such as COVID-19 with serum levels below $11.0 \mathrm{Hb}$ g/L (Ampawong et al., 2015; Ramos, E. M., de Abreu, 2020).

According to Cen et al (2020), hemoglobin levels below $11 \mathrm{~g} / \mathrm{dL}$ are directly related to the worsening of the disease in patients with COVID-19 (Cen et al., 2020; Ramos, E. M., Mendes dos Reis, Ramos, M. V., et al., 2020). In addition, Giacomelli et al. (2020) highlighted in their observational study a high prevalence of patients with COVID-19 and anemia, which totaled 24,262 patients, with the ferritinin level being below the highest standard in populations over the age of 60 years and these patients evolved to death, such evidence confirms the data found in the present study, which is the case report type (International Artemisinin Study Group, 2004; Brenner, Holleczek, \& Schöttker, 2020; Ramos, de Abreu et al., 2020).

Corroborating our findings, Zhou et al. (2020) found in their analysis that the chances of death of patients hospitalized in an intensive care bed are higher among patients with ferritinin levels above $23.9 \mathrm{ng} / \mathrm{mL}$ (Adjuik et al., 2004; Andrade et al., 2010). In fact, this occurs because serum ferritinin is high in patients who progress to death compared to patients recovered over the course of the clinic, and this increase is due to the deterioration of the disease (Ramos, E. M., Mendes dos Reis, Ramos, M. V., et al., 2020). For example, patients with severe hypoxemia had significantly lower serum iron levels than the standard used (Read et al., 2019).

According to Tables 2 and 3, from the assessment of iron quantification in patients with COVID-19 and progression of worsening signs, it was possible to observe that hemoglobin levels decrease with advancing age (Ramos, E. M., de Abreu et al., 2020; Ramos, E. M., Mendes dos Reis, F. J., Ramos, M. V., et al., 2020). In addition, the increase in deaths and morbidities was seen with a greater increase in women, the elderly, diabetics with levels of ferritinin and serum iron below the standard level (Ramos, E. M., de Abreu et al., 2020; Castro et al., 2011).

The serum iron concentration is a determinant of the body's ability to transport oxygen from the blood, thus, in this study it is possible to verify that the serum hemoglobin levels that are lower than the standard established in Brazil, develops the worsening of the patient with COVID-19 mainly in the at-risk population such as the diabetic patients in this study (International Artemisinin Study Group, 2004; Anstey et al., 2002; Ramos, E. M., de Abreu et al., 2020; Ramos, E. M., Mendes dos Reis, F. J., Ramos, M. V., et al., 2020).

Given the above, serum iron can be considered as a biochemical indicator for the worsening of the patient with COVID-19, since the patient with COVID-19 suffers a decrease in the ability of hemoglobin to support the increase in peripheral tissue in the distribution of oxygen in the body, which in turn is linked to the hypermetabolic state during the virus infectious process (Graph 2) (Ampawong et al., 2015; Andrade et al., 2010).

Studies demonstrated that patients with serum iron deficit may develop a change in hemoglobin concentration and develop a decrease in oxygen saturation in the body (Table 2) (International Artemisinin Study Group, 2004; Ampawong et al., 2015). Thus, these patients may worsen the treatment process of COVID-19 and evolve to the need for hospitalization in an intensive care bed due to a decrease in the ability of hemoglobin to develop sepsis in the body with dysfunction of the multiple organ syndrome, usually during the diagnosis period and treatment of the patient with COVID-19 (Ramos, E. M., de Abreu et al., 2020; Ramos, E. M., Mendes dos Reis, F. J., Ramos, M. V., et al., 2020). Viral sepsis is ignored in clinical diagnosis (Ramos, E. M., Mendes dos Reis, F. J., Ramos, M. V., et al., 2020). Therefore, the current evidence in the patient with COVID-19 demonstrates that they develop acute breathing difficulty or acute respiratory distress syndrome, and the prognosis depends on the body's ability to use the demands of peripheral oxygen in the tissues, and when not able to using develops hypoxia and ischemia (Castro et al., 2011; Cooper et al., 2020; Read et al., 2019; Cen et al., 2020).

In view of the above, it can be said that these dysfunctions are related to the serum biochemical marker due to changes in the hemoglobin profile (Gladwin \& Kato, 2008; Ramos, E. M., de Abreu, et al., 2020). Furthermore, it is possible to identify that the worsening of patients with COVID-19 can be compared to the macrophage activation syndrome and that it may be associated with high levels of ferritinin or due to the occurrence of a 
cytokine storm (Ampawong et al., 2015).

Serum iron influences the activation of macrophages in order to increase the secretion of inflammatory cytokines found mainly in patients with COVID-19 (Ramos, E. M., Mendes dos Reis, F. J., Ramos, M. V. et al., 2020). In addition, serum iron can increase ferritin in support of the immune system as an intuition to defend the body against the invading virus (Castro et al., 2011).

The ideal serum iron levels in the host cells are necessary for the defense of the organism by the innate system to occur in an orchestrated way in response to the viral infectious process (Anstey et al., 2002). In fact, the innate system decreases the bioavailability of serum iron in order to decrease the replication of the virus in the acute phase of the infectious process (Ampawong et al., 2015). The increase and storage of serum iron carried out by ferritinin in macrophage cells will help in the fall of serum iron through the increase in serum ferritinin and this process was observed in the results obtained in our study as an acute response (Table 3), which will result in worsening of anemia (Anstey et al., 2002; International Artemisinin Study Group, 2004).

In addition, the worsening of anemia demonstrated a worsening of the main defense cells of patients in the positive control group (Treatment) with COVID-19 (Ampawong et al., 2015; Andrade et al., 2010). Therefore, serum iron and elevated ferritinin in patients with COVID-19 can be used as a screening biomarkers for the severity of inflammation in patients with COVID-19 (Anstey et al., 2002; International Artemisinin Study Group, 2004; Ramos, E. M., Mendes dos Reis, F. J., Ramos, M. V. et al., 2020).

Regarding serum zinc (Table 2 and Graph 2), the data presented in this study highlight the importance of further research in relation to the main role of zinc ( $\mathrm{Zn}$ ) in the immune system (Fawzi et al., 2005). Zinc is an essential microelement for nutrition and plays a role in the physiology of the immune system through activity as a signaling molecule (Ramos, E. M., Mendes dos Reis, et al., 2020; Ramos, E. M., de Abreu, A. C., de Freitas, et al., $2020)^{29,3}$. In addition, this element has specific anti-inflammatory functions in the human body, however, it has antioxidant stabilizing activities on the cell membrane (Fawzi et al., 2005; Prasad et al., 2011; Ramos, E. M., Mendes dos Reis et al., 2020). On the other hand, the deficit of zinc in the human body alters the survival of cells of the immune system by adversely affecting its specific cellular actions, such as phagocytosis, the production of cytokines and the death of target cells (Table 3) (Ramos, E. M., Mendes dos Reis et al., 2020). The zinc deficit causes changes in the mechanism of activation of the response of helper T cells and CD8+ (cytotoxic) T cells (Prasad, 2007; Prasad et al., 2011). Zinc deficiency causes immunodeficiency with the presence of severe lymphopenia which is characterized by a gradual decrease in the development of $\mathrm{B}$ cells in the bone marrow (Ramos, E. M., Mendes dos Reis, et al., 2020; Ramos, E. M., de Abreu, A. C., de Freitas, et al., 2020; Prasad, 2007). Thus, such highlighted evidence is in agreement with the results obtained in the present study (Table 2) (Prasad, 2007; Razzaque, 2020).

In fact, it is due to the zinc deficit that the human body presents a depression in the immune system, and thus, the symptoms of patients with the zinc deficit will worsen and evolve into one of the main differentiating signs in COVID-19 which is the impaired taste and smell (Table 2 and Graph 2) (Ramos, E. M., Mendes dos Reis, Ramos, M. V. et al., 2020; Ramos, E. M., de Abreu, A. C., de Freitas, et al., 2020).

Zinc can protect cells from apoptosis and this action has a protective importance mainly in individuals with diabetes, and in this way zinc potentiates the effect of IFN-agradually in order to neutralize the antagonistic function of IFN by SARS-CoV-2 protein (Ramos, E. M., de Abreu, A. C., de Freitas, et al., 2020; Giacomelli et al., 2020). Thus, it was possible to observe in this study the presence of neutrophilia in patients who evolved with the worsening through the hospitalization process until death (Table 3 and Graph 2) (Giacomelli et al., 2020; Prasad, 2007; D'Avolio et al., 2020). Deficit of zinc causes oxidative damage to phagocytosis in natural killer cells, macrophages and neutrophils (Prasad, 2007). This zinc deficit causes the neutrophil to have difficulty recruiting cells to the site of inflammation and, therefore, undergoes changes in the chemotactic response, which is why we have neutrophilia in the patient diagnosed with COVID-19 (Ramos, E. M., Mendes dos Reis, Ramos, M. V. et al., 2020; Ramos, E. M., Mendes dos Reis, Ramos, M. V. et al., 2020). Therefore, when analyzing the results obtained in this study involving COVID-19, it can be considered that elements such as Zinc are supplemented in patients with COVID-19 being treated, however the serum dosage must be monitored by health professionals such as doctors, nurses and nutritionist on a regular basis during the treatment period (Razzaque, 2020; Read et al., 2019).

In the present study (Table 3 and Graph 2), as seen in the infectious period by COVID-19, zinc levels decreased significantly in patients causing worsening and death (Ramos, E. M., Mendes dos Reis, Ramos, M. V. et al., 2020; Fawzi et al., 2005). In view of the results obtained, this study emphasizes that zinc is effective in immunological protection and useful in zinc deficit known as hypozincemia (serum zinc $<70 \mu \mathrm{g} / \mathrm{dL}$ ) and may decrease the innate 
and adaptive antiviral response (Ramos, E. M., Mendes dos Reis, Ramos, M. V. et al., 2020; Ramos, E. M., de Abreu, A. C. et al., 2020; Fawzi et al., 2005).

Vitamin $\mathrm{D}$ is able to reduce the risk of infectious viral disease as it activates the main physiological actions grouped in forms of physical barrier, as well as cellular in the immune process and adaptive immunity (D'Avolio et al., 2020; Gruber-Bzura, 2018). For example, the respiratory system is considered as the primary site for viral invasion, the deposition of pathogenic microorganisms in the human body, which occurs through inspired air (Ramos, E. M., de Abreu, A. C. et al., 2020; Gruber-Bzura, 2018; Xu et al., 2020). Thus, the respiratory tract brings defenses that involve several factors such as the mucociliary escalator, receptor and effector molecules of the innate immune system (Grant et al., 2020). In this way, this sophisticated defense system is weakened by negative cellular factors capable of aggravating the symptoms of COVID-19, such as age over 60, presence of pre-existing diseases such as diabetes (Table 3) and the deficit of Vitamin D in serum blood (Table 3 and Graph 2) (Grant et al., 2020; Mohammad, Mishra, \& Ashraf, 2019). The actions of vitamin D strengthen immunity through the induction of antimicrobial peptides, such as human catelicidin LL-37 and 1,25-dihydrixivitamin D, which will keep the junction clusters adhering to the gaps in the process of inducing antimicrobial peptide. Deficit of Vitamin D alters the effects of catelicidins, which will exhibit a direct antimicrobial action and decrease the amount of invading microorganisms such as COVID-19 (Prasad, 2007; Razzaque, 2020; Zhou et al., 2020).

The catelicidin has in the human body the function of including and inducing a specific type of pro-inflammatory cytokines that will stimulate the chemotaxis of neutrophils, monocytes, macrophages and Tymphocytes at the site of infection (Ramos, E. M., Mendes dos Reis, Ramos, M. V. et al., 2020; Ramos, E. M., de Abreu, A. C. et al., 2020). Thus, they have the ability to eliminate the virus that causes COVID-19, which is a respiratory pathogen, since it induces apoptosis and autophagy of epithelial cells infected by the COVID-19 virus (Cooper et al., 2020; Gruber-Bzura, 2018). Such processes occur since the 1,25 (OH) D-Vitamin D receptor acts to promote the catelicidin gene, and according to the literature, Vitamin D through this receptor is capable of potentiating the catelicidin transcription. COVID-19 patients activate the innate immune system as a response to the virus and generate pro-inflammation with anti-inflammatory cytokines (Ramos, E. M., Mendes dos Reis, Ramos, M. V. et al., 2020; Ramos, E. M., de Abreu, A. C. et al., 2020). Thus, Vitamin D assists in the treatment of patients with COVID-19, as it reduces the production of pro-inflammatory drugs, mainly auxiliary Cytokines T (Th1), TNF- $\alpha$ and IFN- $\gamma$ with the increase in the expression of anti-inflammatory cytokines by macrophages (Table 3 ) (Ramos, E. M., de Abreu, A. C. et al., 2020).

Vitamin D also promotes the production of cytokines (Th2) mainly by suppressing the production of Th1 cells and this suppression is a complement of immune defense with actions mediated by specific cell types that will induce the production of regulatory T cells (Treg) by inhibition of the inflammatory process (Ramos, E. M., Mendes dos Reis, Ramos, M. V. et al., 2020; Huang et al., 2020).

From the results obtained in the presente study it was possible to verify that the serum concentrations of Vitamin D decrease with increasing age, that is, patients over the age of 60 had a high Vitamin D deficit and this is due to lower sun exposure and levels lower levels develop in patients with less sun exposure time (Ramos, E. M., Mendes dos Reis, Ramos, M. V. et al., 2020; Ramos, E. M., de Abreu, A. C. et al., 2020). In addition, it should be taken into account that the ideal levels of Vitamin D in the blood serum decrease with the use of antiepileptic, antibiotics, anti-inflammatories, antihypertensive, antiretroviral, as well as the use of endocrine and some herbal medicines, and this occurs by activating the pregnane X receptor (Ramos, E. M., Mendes dos Reis, Ramos, M. V. et al., 2020; Ramos, E. M., de Abreu, A. C. et al., 2020). Therefore, the present study (Table 3) is consistent with the results obtained by Gruber-Bzura et al. (2018) in demonstrating that the deficit of Vitamin D reduces the risk of flu-like illnesses such as COVID-19 (Ramos, E. M., Mendes dos Reis, Ramos, M. V. et al., 2020; Mohammad, Mishra, \& Ashraf, 2010).

In terms of the impact on Vitamin D deficit in patients with COVID-19, the present research demonstrated that Vitamin D has a direct relationship in the worsening of complications in COVID-19, because patients with normal blood levels had a slight percentage of worsening (Table 3, Graph 1 and Graph 2) (Mohammad, Mishra, \& Ashraf, 2010; Ramos, E. M., Mendes dos Reis et al., 2020).

Such results prove that the deficit of Vitamin D contributes to the development of the Acute Respiratory Discomfort Syndrome and thus the lethality rates increase with age, comorbidity of chronic disease, being directly associated with a concentration of $1.25(\mathrm{OH})$ lower 2D (Malek Mahdavi, 2020). The normal measurement in blood serum of Vitamin D increases the host's immune response against COVID-19 and its aggressive effects on all organs of the human system. The increase in serum vitamin D levels above $30 \mathrm{ng} / \mathrm{mL}$ has beneficial effects in reducing the incidence and severity of various viral diseases such as COVID-19 (Malek Mahdavi, 2020; Ramos, E. 
M., Mendes dos Reis, Ramos, M. V. et al., 2020).

Patients with a BMI greater than 30 had a high rate of hospital admission due to COVID-19 (Table 1 and Graph 2)

${ }^{31}$. According to results published in the literature. And the COVID-19 enters the human body through the cells of the intestine and alveoli by the dysregulation of the renin-angiotensin system which will contribute to the activation of cytokines and this is deadly in patients who progress to Acute Respiratory Syndrome (Malek Mahdavi, 2020). The increase in obesity (BMI > 30) facilitates the increase in adiposity and affects the internal lung level, such as the alveolar, in which the distribution of immune defense cells can contribute to a maladaptive inflammatory cycle and secondary to the injury process in the body and generally is aggravated by the high presence of hypertension and diabetes mellitus (Malek Mahdavi, 2020). In addition, deficit of Vitamin D contributes to the increase of infections in the respiratory and gastrointestinal tracts (Jin et al., 2013).

The Italian population that died from COVID-19 had a very high prevalence of hypovitaminosis D due to the long winter ${ }^{31}$. Vitamin D has activity in the negative endocrine modulating renin-angiotensin system (RAS) and is able to inhibit renin expression and generation (Grant et al., 2020; Malek Mahdavi, 2020). In addition, Vitamin D is able to reduce the activity of the ACE2/Ang- (1-7)/MasR axis by inhibiting renin on the ACE/Ang II/AT1R axis and thus an increase in the expression and concentration of ACE2 occurs, MasR and Ang- (1-7) that will play a protective role in the potential against acute lung injury in Acute Respiratory Syndrome (Mohammad, Mishra, \& Ashraf, 2010; Grant et al., 2020; Malek Mahdavi, 2020).

According to previous studies (Ramos, E. M., Mendes dos Reis, Ramos, M. V. et al., 2020; Mohammad, Mishra, \& Ashraf, 2010; Jin et al., 2013) and the results observed in this case manuscript, it is possible to identify that Vitamin D can be used as a potential in the therapeutic approach to combat COVID-19.

In addition, the effect of vitamin D against COVID-19 is related to the suppression of the cytokine response and can be used as a biomarker capable of characterizing probabilities of aggravation caused by COVID-19 in populations such as the one investigated in this study and who present with hypovitaminosis D (Table 3 and Graph 2) (Ramos, E. M., Mendes dos Reis, Ramos, M. V. et al., 2020; Mohammad, Mishra, \& Ashraf, 2010; Grant et al., 2020; Malek Mahdavi, 2020).

In addition, Ramos et al. (2020) suggests that hypovitaminosis D may explain geographical variations according to the lethality rates reported in COVID-19, indicating that the deficit in Vitamin D is related to the increase in mortality due to the pandemic. In fact, Vitamin D deficit decreases the TCD4+ lymphocyte count in viral infectious processes like COVID-19 and similarly occurs in HIV infections (Ramos, E. M., Mendes dos Reis, et al., 2020; Ramos, E. M., Mendes dos Reis, F. J., Ramos, M. V. et al., 2020).

In addition, Vitamin D deficiency causes the risk of thrombosis, however, Vitamin D is able to control the expression of several genes relevant to cell proliferation, differentiation, apoptosis and angiogenesis (Mohammad, Mishra, \& Ashraf, 2010).

According to the results obtained in this study, the deficit of Vitamin D increases the risk of death of patients (Graph 1 and Graph 2) and corroborate that the deficit of Vitamin D may be associated with an increase in the worsening of COVID-19, therefore, there is more evidence of the positive role of the use of Vitamin D in the treatment and prevention of aggravations in COVID-19 (Ramos, E. M., Mendes dos Reis, F. J., Ramos, M. V. et al., 2020; Mohammad, Mishra, \& Ashraf, 2010; Jin et al., 2012).

The results of this study are in line with those published by Jakovac et al. (2020), which emphasizes the importance of assessing the status of vitamin D and the polymorphism of the VDR gene in the patient with COVID-19 in order to explain the disproportionate spreading behavior of COVID-19 mainly related to decreased immune functions in individuals with BMI $>30$, that is, the presence of overweight and/or obesity and diabetics related to greater pathogenicity (Table 3 and Graph 1) (Ramos, E. M., de Abreu et al., 2020; Ramos, E. M., Mendes dos Reis, F. J., Ramos, M. V. et al., 2020).

In fact, type II diabetic patients have hyperinsulinemia that will stimulate the reduction of Vitamin D status through sequestration in adipocytes by decreasing the negative charge of the plasma membrane between red blood cells, platelets and endothelial cells that will increase agglutination and cause example thrombosis (Mohammad, Mishra, \& Ashraf, 2010; Cooper et al., 2020; Bishop et al., 2021; Mohammad, Mishra, \& Ashraf, 2019).

\section{Conclusions}

The pandemic caused by COVID-19 in the world and in Brazil has shown a small decrease in cases of death, mainly in Brazil and in the state of Mato Grosso do Sul.

This study brought the possibility of using Zinc, Iron and Vitamin D as elements in order to potentially protect the 
citizens of SARS-CoV-2 or alleviate their illness process by worsening the disease and act as biomarkers for the worsening. The deficiency of Zinc decreases the anti-inflammatory, immunomodulatory and antiviral activities, mainly in COVID-19 and results in the worsening of the disease, as well as the increase in the use of intensive care beds and the death of patients.

As noted, the iron deficit increases the death of patients hospitalized in an intensive care bed, especially in patients with a ferritinin level greater than $23.9 \mathrm{ng} / \mathrm{mL}$. In addition, the level of normal serum iron tends to increase this percentage of ferritinin in the body, giving positive support to the innate immune system in the body's defense process against the invading COVID-19 virus.

Vitamin D has immunomodulatory actions in the body and can have favorable effects on the viral infectious process by COVID-19, and vitamin D deficit increases the risk of death for patients with COVID-19.

\section{Author Contributions}

Conceituação: E.M.R. and V.A.N.; concepção e síntese de compostos, E.M.R. and V.A.N.; Metodologia, E.L.L.A. and F.J.M.d.R.; validação, I.D.d.S., G.G.F.; Investigação, E.M.R., V.A.N, A.C.d.A.; Software, A.C.d.F. and E.M.D.F.; Recursos, V.A.N.; curadoria de dados, E.M.R. and E.L.L.A.; Supervisão, V.A.N.; Administração do projeto, V.A.N and E.M.R.; Visualização, I.B.R and P.A.M.T.

\section{Funding}

This research was financially supported by the the Brazilian Research Council (CNPq) (CNPq: Process No 310621/2020-8) and Coordenação de Aperfeiçoamento de Pessoal de Nível Superior-Brasil (CAPES)-Finance Code 001

\section{Institutional Review Board Statement}

The study was conducted according to the guidelines of the Declaration of Helsinki and approved by the Research Ethics Committee at the Federal University of Mato Grosso do Sul (Reference: CAAE: 42969320.0.0000.0021).

\section{Informed Consent Statement}

Applied to all participants according to Brazilian legislation for conducting research on human beings (Resolution 196/96 of the CNS and improved by Resolution 466/12).

\section{Data Availability Statement}

The data used to support the findings of this study are available from the corresponding author upon request.

\section{Sample Availability}

Samples of the compounds are available from the authors.

\section{Competing Interests Statement}

The authors declare that there are no competing or potential conflicts of interest.

\section{References}

Alves, A., Martins, A., Adolphsson, S., Bockorny, B., Carleti, G., Cabral, G., ... \& Vianna, A. (2007). Severe imported malaria: case report. Revista Brasileira de terapia intensiva, 19(2), 231-236. https://doi.org/10.1590/S0103-507X2007000200016

Ampawong, S., Chaisri, U., Viriyavejakul, P., Prapansilp, P., Grau, G. E., Turner, G. D., \& Pongponratn, E. (2015). A potential role for interleukin-33 and $\gamma$-epithelium sodium channel in the pathogenesis of human malaria associated lung injury. Malaria journal, 14(1), 1-15. https://doi.org/10.1186/s12936-015-0922-x

Andrade, B. B., Reis-Filho, A., Barros, A. M., Souza-Neto, S. M., Nogueira, L. L., Fukutani, K. F., ... \& Barral-Netto, M. (2010). Towards a precise test for malaria diagnosis in the Brazilian Amazon: comparison among field microscopy, a rapid diagnostic test, nested PCR, and a computational expert system based on artificial neural networks. Malaria journal, 9(1), 1-11. PMID: 20459613; PMCID: PMC2883547. https://doi.org/10.1186/1475-2875-9-117

Anstey, N. M., Jacups, S. P., Cain, T., Pearson, T., Ziesing, P. J., Fisher, D. A., ... \& Maguire, G. P. (2002). Pulmonary manifestations of uncomplicated falciparum and vivax malaria: cough, small airways obstruction, impaired gas transfer, and increased pulmonary phagocytic activity. The Journal of infectious diseases, 185(9), 1326-1334. https://doi.org/10.1086/339885

Bishop, L. E., Ismailova, A., Dimeloe, S., Hewison, M., \& White, J. H. (2021). Vitamin D and immune regulation: antibacterial, antiviral, anti-inflammatory. JBMR plus, 5(1), e10405. 
https://doi.org/10.1002/jbm4.10405

Brenner, H., Holleczek, B., \& Schöttker, B. (2020). Vitamin D insufficiency and deficiency and mortality from respiratory diseases in a cohort of older adults: potential for limiting the death toll during and beyond the COVID-19 pandemic?. Nutrients, 12(8), 2488. https://doi.org/10.3390/nu12082488

Castro, T. G. D., Silva-Nunes, M., Conde, W. L., Muniz, P. T., \& Cardoso, M. A. (2011). Anemia and iron deficiency among schoolchildren in the Western Brazilian Amazon: prevalence and associated factors. Cadernos de saude publica, 27(1), 131-142. https://doi.org/10.1590/S0102-311X2011000100014

Cen, Y., Chen, X., Shen, Y., Zhang, X. H., Lei, Y., Xu, C., ... \& Liu, Y. H. (2020). Risk factors for disease progression in patients with mild to moderate coronavirus disease 2019 - a multi-centre observational study. Clinical Microbiology and Infection, 26(9), 1242-1247. https://doi.org/10.1016/j.cmi.2020.05.041

Chan, J. F. W., Yuan, S., Kok, K. H., To, K. K. W., Chu, H., Yang, J., ... \& Yuen, K. Y. (2020). A familial cluster of pneumonia associated with the 2019 novel coronavirus indicating person-to-person transmission: a study of a family cluster. The lancet, 395(10223), 514-523. PubMed PMID: 31986261. Epub 2020/01/28. eng. 2020 .

Cooper, I. D., Crofts, C. A., DiNicolantonio, J. J., Malhotra, A., Elliott, B., Kyriakidou, Y., \& Brookler, K. H. (2020). Relationships between hyperinsulinaemia, magnesium, vitamin D, thrombosis and COVID-19: rationale for clinical management. Open Heart, 001356. https://doi.org/10.1136/openhrt-2020-001356

D’Avolio, A., Avataneo, V., Manca, A., Cusato, J., De Nicolò, A., Lucchini, R., ... \& Cantù, M. (2020). 25-Hydroxyvitamin D concentrations are lower in patients with positive PCR for SARS-CoV-2. Nutrients, 12(5), 1359. https://doi.org/10.3390/nu12051359

Fawzi, W. W., Villamor, E., Msamanga, G. I., Antelman, G., Aboud, S., Urassa, W., \& Hunter, D. (2005). Trial of zinc supplements in relation to pregnancy outcomes, hematologic indicators, and $\mathrm{T}$ cell counts among HIV-1-infected women in Tanzania. The American journal of clinical nutrition, 81(1), 161-167. https://doi.org/10.1093/ajcn/81.1.161

Giacomelli, A., Ridolfo, A. L., Milazzo, L., Oreni, L., Bernacchia, D., Siano, M., ... \& Galli, M. (2020). 30-day mortality in patients hospitalized with COVID-19 during the first wave of the Italian epidemic: a prospective cohort study. Pharmacological research, $158, \quad 104931$. https://doi.org/10.1016/j.phrs.2020.104931

Gladwin, M. T., \& Kato, G. J. (2008). Hemolysis-associated hypercoagulability in sickle cell disease: the plot (and blood) thickens!. Haematologica, 93(1), 1. https://doi.org/10.3324/haematol.12318

Grant, W. B., Lahore, H., McDonnell, S. L., Baggerly, C. A., French, C. B., Aliano, J. L., \& Bhattoa, H. P. (2020). Evidence that vitamin D supplementation could reduce risk of influenza and COVID-19 infections and deaths. Nutrients, 12(4), 988. https://doi.org/10.3390/nu12040988

Gruber-Bzura, B. M. (2018). Vitamin D and influenza-prevention or therapy?. International journal of molecular sciences, 19(8), 2419. https://doi.org/10.3390/ijms19082419

Huang, Y., Tu, M., Wang, S., Chen, S., Zhou, W., Chen, D., ... \& Guo, L. (2020). Clinical characteristics of laboratory confirmed positive cases of SARS-CoV-2 infection in Wuhan, China: A retrospective single center analysis. Travel medicine and infectious disease, 36, 101606. https://doi.org/10.1016/j.tmaid.2020.101606

International Artemisinin Study Group. (2004). Artesunate combinations for treatment of malaria: meta-analysis. The Lancet, 363(9402), 9-17. https://doi.org/10.1016/S0140-6736(03)15162-8

Jin, H. J., Lee, J. H., \& Kim, M. K. (2013). The prevalence of vitamin D deficiency in iron-deficient and normal children under the age of 24 months. Blood research, 48(1), 40-45. https://doi.org/10.5045/br.2013.48.1.40

Malek Mahdavi, A. (2020). A brief review of interplay between vitamin D and angiotensin-converting enzyme 2: Implications for a potential treatment for COVID-19. Reviews in Medical Virology, 30(5), e2119. PMID: 32584474. PMCID: PMC7362103. https://doi.org/10.1002/rmv.2119

Mohammad, S., Mishra, A., \& Ashraf, M. Z. (2019). Emerging role of vitamin D and its associated molecules in pathways related to pathogenesis of thrombosis. Biomolecules, 9(11), 649. https://doi.org/10.3390/biom9110649 
OPAS, OMS. Folha informativa - COVID-19 (doença causada pelo novo coronavírus). (2020). Retrieved from https://www.paho.org/bra/index.php?option=com_content\&view=article\&id=6101:covid19\&Itemid=875

Prasad, A. S. (2007). Zinc: mechanisms of host defense. The Journal of nutrition, 137(5), 1345-1349. https://doi.org/10.1093/jn/137.5.1345

Prasad, A. S., Bao, B., Beck, F. W., \& Sarkar, F. H. (2011). Zinc-suppressed inflammatory cytokines by induction of A20-mediated inhibition of nuclear factor-kB. Nutrition, 27(7-8), 816-823. https://doi.org/10.1016/j.nut.2010.08.010

Ramos, E. M., de Abreu, A. C., de Freitas, S. L. F., de Lima, M. D., dos Reis, F. J. M., Ramos, H. V., ... \& do Nascimento, V. A. (2020). COVID-19, rate of case factors and nutritional characteristics of patients dying in Italy and Brazil: a critical analyze. Global J Health Sci, 12(7), 133. https://doi.org/10.5539/gjhs.v12n7p133

Ramos, E. M., Mendes dos Reis, F. J., de Souza, I. D., de Freitas, S. L. F., de Lima, M. D., \& de Abreu, A. C. (2020). Does Vitamin D Supplementation have a Positive and Important Response in the Immune System on Covid-19 Pandemic? A Short Critical Analysis. International Journal, 8(1), 33-39. https://doi.org/10.15640/ijmp.v8n1a5

Ramos, E. M., Mendes dos Reis, F. J., Ramos, M. V., de Souza, I. D., \& Bochenek, L. D. S. (2020). Vitamin D produce antibodies in pandemic response to gripal viruses? A critical analysis. Int J Clin Virol, 4, 023-026. https://doi.org/10.29328/journal.ijcv.1001010

Razzaque, M. S. (2020). COVID-19 pandemic: can maintaining optimal zinc balance enhance host resistance? The Tohoku journal of experimental medicine, 251(3), 175-181. PMID: 32641644. https://doi.org/10.1620/tjem.251.175

Read, S. A., Obeid, S., Ahlenstiel, C., \& Ahlenstiel, G. (2019). The role of zinc in antiviral immunity. Advances in nutrition, 10(4), 696-710. https://doi.org/10.1093/advances/nmz013

Xu, T., Huang, R., Zhu, L., Wang, J., Cheng, J., Zhang, B., ... \& Liu, L. (2020). Epidemiological and clinical features of asymptomatic patients with SARS-CoV-2 infection. Journal of medical virology, 92(10), 1884-1889. https://doi.org/10.1002/jmv.25944

Zhou, F., Yu, T., Du, R., Fan, G., Liu, Y., Liu, Z., ... \& Cao, B. (2020). Clinical course and risk factors for mortality of adult inpatients with COVID-19 in Wuhan, China: a retrospective cohort study. The lancet, 395(10229), 1054-1062. https://doi.org/10.1016/S0140-6736(20)30566-3

\section{Copyrights}

Copyright for this article is retained by the author(s), with first publication rights granted to the journal.

This is an open-access article distributed under the terms and conditions of the Creative Commons Attribution license (http://creativecommons.org/licenses/by/4.0/). 